\title{
Board Stiff TEE Transesophageal Echocardiography - Second Edition
}

\author{
Christopher J. Gallagher, John C. Sciarra, Steven Ginsberg. Elsevier Saunders, \\ New York, 2013. ExpertConsult.com. ISBN: 978-1-4557-3805-2
}

\author{
Benjamin Sohmer, MD
}

Received: 24 June 2013/Accepted: 8 July 2013/Published online: 19 July 2013

(C) Canadian Anesthesiologists' Society 2013

Board Stiff TEE Transesophageal Echocardiography provides a simplified introduction to the field of perioperative transesophageal echocardiography (TEE). This second edition textbook of roughly 300 pages is an updated version of the original 2004 publication with the addition of new pertinent perioperative TEE topics, including three-dimensional (3D) TEE. Sonographic formulas and sample calculations have also been amalgamated into concise straightforward chapters. This book will serve potential learners who wish to explore and gain knowledge about perioperative TEE. The text provides a starting point from which learners can embark on what many may consider to be a difficult task. Given its likely readership, the book's title and cover are somewhat misleading; as mentioned in the preface, this book was not written as a reference text for either board exam preparation or as a review tool.

The textbook offers many benefits. To begin with, TEE language is decoded. By providing the reader an introduction to the lingo of echocardiography, the learner can better understand other educational echocardiography resources. The layout of the chapters is logical; it follows the same subject headings as other TEE references and the PTEeXAM subject list. This textbook is a great starting point for novice TEE learners beginning a self-directed learning path. Furthermore, this text has the potential of being very beneficial for those who are not enrolled in a formal fellowship program, as it is written in a style that mimics typical intraoperative bedside teaching. The chapters are written to entertain like the script for a lecture. In a field where the material can potentially be dry

B. Sohmer, MD $(\bowtie)$

University of Ottawa, Ottawa, ON, Canada

e-mail: bsohmer@ottawaheart.ca and dull, this textbook is a light read that can maintain the attention of readers as they go over the material. As TEE knowledge is expected to be disseminated beyond the cardiac anesthesiologist and fellow, the text undoubtedly provides non-cardiac anesthesiologists who may contemplate embarking on this learning with an excellent overview of the overall scope of TEE knowledge. Two highlights of the book are the basics of ultrasound technology and the section on artifacts and pitfalls. With both topics, the authors take complex subjects and provide a proficient foundation of understanding.

There are several drawbacks to this text beginning with the title. By no means should this text be used as a primary resource for "board" exam preparation. It is not comprehensive and does not provide adequate in-depth knowledge of any TEE subject. As mentioned above, the text may be beneficial to those who want to get their feet wet, but it serves little role beyond that, particularly for cardiac anesthesia fellows who have access to established TEE training curricula. Though amusing at first, the text contains too many forced jokes that potentially distract from the subject matter. Not everyone will appreciate the humour, and readers may in fact be turned off by the frivolity of the prose. In addition, in this age of advanced multimedia, the illustrations and diagrams that are depicted are dated and too simplistic. Echocardiography is a visual science that is based on image acquisition and interpretation. Presenting the equivalent of hand-drawn schematics just doesn't measure up to the expected graphic standards of 2013. Lastly, the textbook fails to relay an important concept to the novice learner, namely, how two-dimensional (2D) images are created from a 3D live heart. One of the most difficult tasks to learn is how to create a mental reproduction of the heart using 2D images. This text does not address this issue optimally. 
When putting together a book such as this, it should be recognized that there is a fine balance between what to include in the contents and what to leave out. Complex TEE topics have no real place in this text. It is this reviewer's opinion, however, that a 3D chapter should be included in any new edition of a TEE text. This text's 3D chapter is too simple for anyone who is familiar with 3D, yet it is too complex for a novice echocardiographer.
All in all, this book is an excellent place to start for those unfamiliar with echocardiography or those who simply want to learn the language of echo, especially if they are not in a formal TEE training program.

Conflicts of interest None declared. 